\title{
ON TCHEBYSHEFF SYSTEMS
}

\author{
KAZUAKI KITAHARA
}

(Communicated by Richard R. Goldberg)

\begin{abstract}
Let $u_{1}, \ldots, u_{n}$ be linearly independent continuously differentiable functions on the unit interval. In this paper, we obtain the following two results. One is a necessary and sufficient condition for the span of $\left\{1, u_{1}, \ldots, u_{n}\right\}$ to have a Markoff basis containing 1 . The other is that any Markoff system $\left\{u_{i}\right\}_{i=1}^{n}$ has a Tchebysheff extension $u_{n+1}$ which is continuously differentiable.
\end{abstract}

\section{INTRODUCTION}

Let $u_{1}, \ldots, u_{n}$ denote linearly independent functions in $C[0,1]$, the space of all real-valued continuous functions on the closed unit interval $[0,1]$. Then $\left\{u_{i}\right\}_{i=1}^{n}$ is said to be a Tchebysheff system (respectively a weak Tchebysheff system) if every nonzero function in the linear subspace $L\left[u_{1}, \ldots, u_{n}\right]$ spanned by $\left\{u_{i}\right\}_{i=1}^{n}$ has no more than $n-1$ zeros (respectively changes of sign) in $[0,1]$. For brevity, a Tchebysheff system (respectively a weak Tchebysheff system) is called a $T$-system (respectively a $W T$-system) and the linear subspace $L\left[u_{1}, \ldots, u_{n}\right]$ called a $T$-space (respectively a $W T$-space).

As is well known, $T$-systems and $W T$-systems are of great use in considering best approximation problems with the uniform norm or the $L^{1}$-norm, and many important properties of these systems have been obtained (see [1, 2 and 5-10]).

The purpose of this paper is to show the following two results of a system of continuously differentiable functions $\left\{u_{i}\right\}_{i=1}^{n}$. One is a necessary and sufficient condition for the span of $\left\{1, u_{1}, \ldots, u_{n}\right\}$ to have a Markoff basis containing 1 . The other is that, for any Markoff system $\left\{u_{i}\right\}_{i=1}^{n}$ on a closed interval, there is a Tchebysheff extension $u_{n+1}$ of $\left\{u_{i}\right\}_{i=1}^{n}$ which is continuously differentiable, i.e., there exists a $u_{n+1}$ of $C^{1}[0,1]$ such that $\left\{u_{i}\right\}_{i=1}^{n+1}$ is a $T$-system. To do them, we pay attention to a subclass of $W T$-systems which does not contain the spline spaces but contains the $T$-systems and study some properties of the systems of this subclass. For the sake of convenience, we name this system,

Received by the editors September 14, 1987.

1980 Mathematics Subject Classification (1985 Revision). Primary 41A50.

Key words and phrases. Weak Tchebysheff systems, Tchebysheff systems. 
which is defined in $\S 1$, an integral Tchebysheff system or an IT-system and call the linear subspace spanned by an $I T$-system an $I T$-space.

\section{IT-SYSTEMS}

First we make the following preparations: By a subinterval of $[0,1]$ we mean a nondegenerate one and a set $\left\{I_{\lambda}\right\}_{\lambda \in \Lambda}$ of subintervals of $[0,1]$ is called disjoint if every $I_{\lambda} \cap I_{\lambda^{\prime}}, \lambda \neq \lambda^{\prime}$, is a degenerate interval or empty. For a given positive integer $n$, we set $A_{n}=\left\{\left(t_{1}, \ldots, t_{n}\right) \mid 0<t_{1}<\cdots<t_{n}<1\right\}$, and for a function $u$ of $C[0,1]$, the set of zeros of $u$ is denoted by $Z(u)$. Let $u_{1}, \ldots, u_{n}$ be functions in $C[0,1]$ and $t_{1}, \ldots, t_{n}$ points in $[0,1]$. Then we denote the $n$th order determinant by

$$
D\left(\begin{array}{c}
u_{1}, \ldots, u_{n} \\
t_{1}, \ldots, t_{n}
\end{array}\right)=\left|\begin{array}{cc}
u_{1}\left(t_{1}\right), \ldots, u_{n}\left(t_{n}\right) \\
\vdots & \vdots \\
u_{1}\left(t_{1}\right), \ldots, u_{n}\left(t_{n}\right)
\end{array}\right|
$$

Now we give the definition of $I T$-systems.

Definition 1. Let $\left\{u_{i}\right\}_{i=1}^{n}$ be linearly independent functions in $C[0,1]$. Then $\left\{u_{i}\right\}_{i=1}^{n}$ is said to be an $I T$-system if for any disjoint $n$-subintervals $I_{i}, i=$ $1, \ldots, n$, of $[0,1]$, the $n$th order determinant

Then we have

$$
\left|\begin{array}{cc}
\int_{I_{1}} u_{1} d x, \ldots, \int_{I_{n}} u_{1} d x \\
\vdots & \vdots \\
\int_{I_{1}} u_{n} d x, \ldots, \int_{I_{n}} u_{n} d x
\end{array}\right| \neq 0
$$

Theorem 1. For linearly independent functions $\left\{u_{i}\right\}_{i=1}^{n}$ in $C[0,1]$, the following conditions are equivalent:

(1) $\left\{u_{i}\right\}_{i=1}^{n}$ is an IT-system.

(2) $\left\{u_{i}\right\}_{i=1}^{n}$ is a WT-system and, for any $f \in L\left[u_{1}, \ldots, u_{n}\right]-\{0\}, Z(f)$ is nowhere dense in $[0,1]$.

(3) $D\left(\begin{array}{c}\sigma u_{1}, \ldots, u_{n} \\ t_{1}, \ldots, t_{n}\end{array}\right) \geq 0$ for all $\left(t_{1}, \ldots, t_{n}\right) \in A_{n}$, where $\sigma=1$ or -1 , and $B_{n}\left(\sigma u_{1}, \ldots, u_{n}\right)=\left\{\left(t_{1}, \ldots, t_{n}\right) \mid\left(t_{1}, \ldots, t_{n}\right) \in A_{n}, D\left(\begin{array}{c}\sigma u_{1}, \ldots, u_{n} \\ t_{1}, \ldots, t_{n}\end{array}\right)>0\right\}$ is dense in $A_{n}$.

Proof. $(1) \Rightarrow(2)$. Suppose that $\left\{u_{i}\right\}_{i=1}^{n}$ is not a $W T$-system. Then there is a function $f \in L\left[u_{1}, \ldots, u_{n}\right]-\{0\}$ such that, for some $0<t_{1}<\cdots<t_{n+1}<1$,

$$
f\left(t_{i}\right) \cdot f\left(t_{i+1}\right)<0, \quad i=1, \ldots, n .
$$

By (1.1), there exist disjoint subintervals $I_{i} \subset\left[t_{i}, t_{i+1}\right], i=1,2, \ldots, n$, such that

$$
\int_{I_{i}} f d x=0, \quad i=1, \ldots, n .
$$

But (1.2) contradicts the definition of $I T$-systems. Next suppose that, for some $g \in L\left[u_{1}, \ldots, u_{n}\right]-\{0\}, Z(g)$ is not nowhere dense in $[0,1]$. From this 
assumption, we can easily find disjoint subintervals $I_{i}, i=1, \ldots, n$, such that $g$ vanishes identically on each $I_{i}$. This is also contradictory to the fact that $\left\{u_{i}\right\}_{i=1}^{n}$ is an $I T$-system.

(2) $\Rightarrow(1)$. If $\left\{u_{i}\right\}_{i=1}^{n}$ is not an IT -system, then, for some $f \in L\left[u_{1}, \ldots, u_{n}\right]$ - $\{0\}$ and some disjoint subintervals $I_{i}, i=1, \ldots, n$, of $[0,1]$,

$$
\int_{I_{i}} f d x=0, \quad i=1, \ldots, n \text {. }
$$

Since $f$ does not vanish identically on each $I_{i}$, from the continuity of $f$, and (1.3), we obtain

$$
f\left(t_{i}\right) \cdot f\left(s_{i}\right)<0 \text { for some } t_{i}, s_{i} \in I_{i}, i=1, \ldots, n .
$$

This implies that $\left\{u_{i}\right\}_{i=1}^{n}$ is not a $W T$-system.

(1) $\Rightarrow(3)$. For $I_{1} \leq \cdots \leq I_{n}$ (i.e., $I_{1} \times \cdots \times I_{n} \subset A_{n}$ ), applying problem 68 , p. 61, in Pólya and Szegö [3], we see that

$$
\begin{aligned}
& \operatorname{det}\left(\int_{I_{j}} u_{i}(x) d x\right)_{i, j=1}^{n}=\operatorname{det}\left(\int_{[0,1]} u_{i}(x) \chi_{I_{j}}(x) d x\right)_{i, j=1}^{n} \\
& \quad=\frac{1}{n !} \int_{0<x_{1}<\cdots<x_{n}<1} \cdots \int_{\operatorname{lot}\left(u_{i}\left(x_{j}\right)\right)_{i, j=1}^{n} \operatorname{det}\left(\chi_{I_{i}}\left(x_{j}\right)\right)_{i, j=1}^{n} d x_{1} \cdots d x_{n}} \\
& =\frac{1}{n !} \int_{I_{n}} \cdots \int_{I_{1}} \operatorname{det}\left(u_{i}\left(x_{j}\right)\right)_{i, j=1}^{n} d x_{1} \cdots d x_{n},
\end{aligned}
$$

where each $\chi_{I_{j}}(x), 1 \leq j \leq n$, is the characteristic function of $I_{j}$. Then (3) $\Rightarrow$ (1) follows immediately from (1.4). By (1) $\Leftrightarrow(2)$ and (1.4), if

$$
\operatorname{det}\left(u_{i}\left(x_{j}\right)\right)_{i, j=1}^{n}=0
$$

on an open subset of $A_{n}$, we can easily choose $I_{1} \leq \cdots \leq I_{n}$ contained in $[0,1]$ whose product is in this open set and thus $\left(\int_{I_{j}} u_{i}(x) d x\right)_{i, j=1}^{n}=0$. This implies that $(1) \Rightarrow(3)$ holds.

Remark 1. (1) As a typical example of an $I T$-system, let $\left\{u_{i}\right\}_{i=1}^{n}$ be a $T$-system and $w$ a nonnegative continuous function such that $Z(w)$ is nowhere dense in $[0,1]$, then the system $\left\{w u_{i}\right\}_{i=1}^{n}$ is an IT-system.

(2) In the rest of this paper, without loss of generality, we assume that, for a $W T$-system $\left\{u_{i}\right\}_{i=1}^{n}$,

$$
D\left(\begin{array}{c}
u_{1}, \ldots, u_{n} \\
t_{1}, \ldots, t_{n}
\end{array}\right) \geq 0, \quad\left(t_{1}, \ldots, t_{n}\right) \in A_{n} .
$$

\section{BASIC PROPERTIES OF IT -SYSTEMS}

We begin by stating the following theorem regarding $W T$-spaces.

Theorem A (Sommer and Strauss [6] and Stockenberg [7]). Let $U$ be an $n$ dimensional WT-space; then there exists an $(n-1)$-dimensional WT-subspace of $U$.

In case of $I T$-spaces, we prove 
Theorem 2. Let $U$ be an n-dimensional IT-space; then there exists an (n-1)dimensional IT-subspace of $U$.

Proof. By Theorem 1, $U$ is a $W T$-space, and using Theorem A, $U$ contains an $(n-1)$-dimensional $W T$-subspace $U_{0}$ of $U$. Since, for any $f \in U_{0}-\{0\}$, $Z(f)$ is nowhere dense in $[0,1], U_{0}$ is an $I T$-subspace by Theorem 1 .

Remark 2. As a result of Theorem 2, we observe that every $n$-dimensional ITspace has a basis $\left\{u_{i}\right\}_{i=1}^{n}$ such that each system $\left\{u_{i}\right\}_{i=1}^{j}, 1 \leq j \leq n$, is an IT -system.

Let us recall that, for a system $\left\{u_{i}\right\}_{i=1}^{n}$, the convexity cone $K\left[u_{1}, \ldots, u_{n}\right]$ is the set of all real-valued functions $f$ defined on $(0,1)$ for which the determinant

$$
D\left(\begin{array}{c}
u_{1}, \ldots, u_{n}, f \\
t_{1}, \ldots, t_{n}, t_{n+1}
\end{array}\right) \geq 0 \text { for all }\left(t_{1}, \ldots, t_{n+1}\right) \in A_{n+1} .
$$

Furthermore we denote by $K_{c}\left[u_{1}, \ldots, u_{n}\right]$ the set of all functions in $K\left[u_{1}, \ldots, u_{n}\right]$ which are continuous on $[0,1]$.

Zielke [9] and Zalik [8] proved that, for a $T$-system $\left\{u_{i}\right\}_{i=1}^{n}$, there is a $u_{n+1}$ in $K_{c}\left[u_{1}, \ldots, u_{n}\right]$ such that $\left\{u_{i}\right\}_{i=1}^{n+1}$ is a $T$-system.

We shall prove the similar result for an $I T$-system. First, we need the following

Lemma. Let $\left\{u_{i}\right\}_{i=1}^{n}$ be an IT-system. Suppose that there exist a countable dense subset $S=\left\{\left(s_{1}^{(i)}, \ldots, s_{n}^{(i)}\right), i \in N\right\}$ of $A_{n}$ and a sequence $\left\{f_{i}\right\}_{i \in N}$ of functions in $K_{c}\left[u_{1}, \ldots, u_{n}\right]$ such that, for every $\left(s_{1}^{(i)}, \ldots, s_{n}^{(i)}\right)$ and $f_{i}$,

$$
\left\{t \mid D\left(\begin{array}{l}
u_{1}, \ldots, u_{n}, f_{i} \\
s_{1}^{(i)}, \ldots, s_{n}^{(i)}, t
\end{array}\right)>0, t \in\left(s_{n}^{(i)}, 1\right)\right\}
$$

is dense in the open interval $\left(s_{n}^{(i)}, 1\right)$. Then there is a $u_{n+1}$ in $K_{c}\left[u_{1}, \ldots, u_{n}\right]$ such that $\left\{u_{i}\right\}_{i=1}^{n+1}$ is an IT-system.

Proof. Setting $u_{n+1}(t)=\sum_{i=1}^{\infty} 2^{-i}\left\|f_{i}\right\|^{-1} \cdot f_{i}(t)$, where $\|\cdot\|$ denotes the uniform norm on $[0,1], u_{n+1}$ is clearly contained in $K_{c}\left[u_{1}, \ldots, u_{n}\right]$ and the subset $\left\{\left(t_{1}, \ldots, t_{n}\right) \mid D\left(u_{1}, \ldots, u_{n+1}\right)>0,\left(t_{1}, \ldots, t_{n+1}\right) \in A_{n+1}\right\}$ is dense in $A_{n+1}$. Hence, from Theorem 1, the conclusion follows immediately.

Now we show

Theorem 3. If $\left\{u_{i}\right\}_{i=1}^{n}$ is an IT-system, there is a $u_{n+1}$ in $K_{c}\left[u_{1}, \ldots, u_{n}\right]$ such that $\left\{u_{i}\right\}_{i=1}^{n+1}$ is an IT-system.

Proof. In case $n=1$, by setting $u_{2}(t)=t \cdot u_{1}(t) ;\left\{u_{1}, u_{2}\right\}$ is an IT-system.

In case $n \geq 2$, by Remark 2 , we assume that each system $\left\{u_{i}\right\}_{i=1}^{k}, 1 \leq k \leq$ $n$, is an $I T$-system. Since, by Theorem $1, B_{n}\left(u_{1}, \ldots, u_{n}\right)$ and $\{(t, s) \mid(t, s) \in$ $\left.A_{n}, s \in B_{n-1}\left(u_{1}, \ldots, u_{n-1}\right)\right\}$ are open and dense in $A_{n}$, we can take a countable dense subset $S=\left\{\left(s_{1}^{(i)}, \ldots, s_{n}^{(i)}\right), i \in N\right\}$ in $A_{n}$ such that

$$
D\left(\begin{array}{c}
u_{1}, \ldots, u_{n} \\
s_{1}^{(i)}, \ldots, s_{n}^{(i)}
\end{array}\right)>0 \text { for } i \in N
$$


and

$$
D\left(\begin{array}{c}
u_{1}, \ldots, u_{n-1} \\
s_{2}^{(i)}, \ldots, s_{n}^{(i)}
\end{array}\right)>0 \text { for } i \in N
$$

For every $\left(s_{1}^{(i)}, \ldots, s_{n}^{(i)}\right) \in S$, using the same method as the proof of Theorem 1 in Zalik [8], we find a $V(t) \in K_{c}\left[u_{1}, \ldots, u_{n}\right]$ such that

$$
V(t)= \begin{cases}0, & t \in\left[0, s_{n}^{(i)}\right], \\ \sum_{i=1}^{n} a_{i} u_{i}(t), & t \in\left[s_{n}^{(i)}, 1\right], \text { where } a_{n}=1\end{cases}
$$

Hence putting

$$
\begin{array}{r}
f(t)=D\left(\begin{array}{c}
u_{1}, \ldots, u_{n}, V \\
s_{1}^{(i)}, \ldots, s_{n}^{(i)}, t
\end{array}\right)=V(t) \cdot D \\
\left(\begin{array}{c}
u_{1}, \ldots, u_{n} \\
s_{1}^{(i)}, \ldots, s_{n}^{(i)}
\end{array}\right) \\
\text { for } t \in\left[s_{n}^{(i)}, 1\right],
\end{array}
$$

by (2.1), (2.2) and (2.3), $f$ is nonnegative and contained in $\left.L\left[u_{1}, \ldots, u_{n}\right]\right|_{\left[s_{n}^{(i)}, 1\right]}$ $-\{0\}$, where $\left.L\left[u_{1}, \ldots, u_{n}\right]\right|_{\left[s_{n}^{(i)}, 1\right]}$ denotes the linear space obtained by restricting $L\left[u_{1}, \ldots, u_{n}\right]$ to $\left[s_{n}^{(i)}, 1\right]$. Since $\left\{u_{i}\right\}_{i=1}^{n}$ is an $I T$-system, $\{t \mid f(t)>0$, $\left.t \in\left(s_{n}^{(i)}, 1\right)\right\}$ is dense in $\left(s_{n}^{(i)}, 1\right)$ by'Theorem 1 . Thus the condition of Lemma holds.

\section{MAIN THEOREMS}

In the first place, we need the following

Definition 2. (1) Let $\left\{u_{i}\right\}_{i=1}^{n}$ be a $T$-system (respectively a $W T$-system). If each system $\left\{u_{i}\right\}_{i=1}^{j}, 1 \leq j \leq n$, is a $T$-system (respectively a $W T$-system), then $\left\{u_{i}\right\}_{i=1}^{n}$ is said to be a Markoff system (respectively a weak Markoff system).

(2) For an $n$-dimensional linear subspace $U$ of $C[0,1]$, a basis $\left\{u_{i}\right\}_{i=1}^{n}$ of $U$ is called a Markoff basis (respectively a weak Markoff basis) if it is a Markoff system (respectively a weak Markoff system).

Providing that $U$ is an $n(\geq 2)$-dimensional linear subspace of continuously differentiable functions, containing constants, Zwick [10] has given the following characterization of $U$ having a weak Markoff basis.

Theorem B. Let $U$ be an n-dimensional subspace of $C^{1}[0,1]$ which contains constants. Then $U$ has a weak Markoff basis $\left\{u_{i}\right\}_{i=1}^{n}$ with $u_{1}=1$ if and only if the space of derivatives $U^{\prime}$ is a WT-space.

Replacing a weak Markoff basis with a Markoff basis, we obtain

Theorem 4. Let $U$ be an n-dimensional subspace of $C^{1}[0,1]$ which contains constants. Then $U$ has a Markoff basis $\left\{u_{i}\right\}_{i=1}^{n}$ with $u_{1}=1$ if and only if the space of derivatives $U^{\prime}$ is an IT-space.

Proof. Suppose that $U$ has a Markoff basis $\left\{u_{i}\right\}_{i=1}^{n}$ with $u_{1}=1$. Since, by Theorem $\mathrm{B}, U^{\prime}$ is a $W T$-space, applying Theorem 1 , it is sufficient to show that 
$Z(f)$ is nowhere dense in $[0,1]$ for every $f \in U^{\prime}=\{0\}$. To do this, assume that a nonzero function $f=\sum_{i=1}^{n} a_{i} u_{i}^{\prime}$ vanishes identically on a subinterval $[a, b]$ of $[0,1]$. Then the function $\sum_{i=1}^{n} a_{i} u_{i}$ is equal to a constant on $[a, b]$. But this contradicts the fact that $U$ has a Markoff basis.

Conversely suppose that $U^{\prime}$ is an $I T$-space. From Theorem 1 and Theorem B, we have a weak Markoff basis $\left\{u_{i}\right\}_{i=1}^{n}$ with $u_{1}=1$ of $U$. Furthermore we shall show that each system $\left\{u_{i}\right\}_{i=1}^{j}, 2 \leq j \leq n$, is a $T$-system. As in the first half of this proof, suppose that a function $g=\sum_{i=1}^{j} b_{i} u_{i} \in L\left[u_{1}, \ldots, u_{n}\right]-\{0\}$ vanishes identically on a subinterval $[c, d]$ of $[0,1]$. Since $b_{2}^{2}+\cdots+b_{j}^{2} \neq 0$, $g^{\prime}=\sum_{i=2}^{j} b_{i} u_{i}^{\prime}$ is contained in $U^{\prime}-\{0\}$ and vanishes identically on $[c, d]$. But this is contradictory to the assumption on $U^{\prime}$. Hence applying Theorem 2.45 in Schumaker [4], we can conclude tht each $\left\{u_{i}\right\}_{i=1}^{j}$ is a $T$-system on the open interval $(0,1)$. By Theorem $\mathrm{B},\left\{u_{i}^{\prime}\right\}_{i=2}^{j}$ is a $W T$-system on $[0,1]$. If $u$ in $L\left[u_{1}, \ldots, u_{j}\right]-\{0\}$ has $j$ zeros in $[0,1]$, then between each pair of zeros $u^{\prime}$ attains positive and negative values and hence has $j$ points of sign change. This is a contradiction. Thus each system $\left\{u_{i}\right\}_{i=1}^{j}, 2 \leq j \leq n$, is a $T$-system on $[0,1]$.

Theorem 5. If $\left\{u_{i}\right\}_{i=1}^{n}$ is a Markoff system consisting of continuously differential functions, then there exists a continuously differentiable function $u_{n+1}$ such that $\left\{u_{i}\right\}_{i=1}^{n+1}$ is a Markoff system.

Proof. Since $\left\{u_{i}\right\}_{i=1}^{n}$ is a Markoff system on $[0,1], u_{1}$ does not vanish on $[0,1]$. By setting $v_{i}=u_{i} / u_{1}, 1 \leq i \leq n$, we can reduce to a Markoff system $\left\{v_{i}\right\}_{i=1}^{n}$ with $v_{1}=1$. By Theorem $4, v_{2}^{\prime}, \ldots, v_{n}^{\prime}$ is an $I T$-system. Then, using Theorem 3, we have such an $f$ of $C[0,1]$ that $\left(v_{2}^{\prime}, \ldots, v_{n}^{\prime}, f\right)$ is an ITsystem. Hence, setting $v_{n+1}(t)=\int_{0}^{t} f(x) d x$, we easily observe that $\left\{v_{i}\right\}_{i=1}^{n+1}$ is a Markoff system by Theorem 4 .

\section{ACKNOWLEDGMENT}

The author wishes to express his gratitude to the referee who gave him many valuable comments on this article.

\section{REFERENCES}

1. M. W. Bartelt, Weak Chebyshev sets and splines, J. Approx. Theory 14 (1975), 30-37.

2. R. C. Jones and L. A. Karlovitz, Equioscillation under nonuniqueness in the approximation of continuous functions, J. Approx. Theory 3 (1970), 138-145.

3. G. Pólya and G. Szegö, Problems and theorems in analysis. I, Springer-Verlag, New York, 1972.

4. L. Schumaker, Spline functions: Basic theory, Wiley-Interscience, New York, 1981.

5. M. Sommer, Weak Chebyshev spaces and best $L^{\prime}$-approximation, J. Approx. Theory 39 (1983), 54-71.

6. M. Sommer and H. Strauss, Eigenschaften von schwach Tschebyscheffschen Räumen, J. Approx. Theory 21 (1977), 257-268. 
7. B. Stockenberg, Subspaces of weak and oreinted Tchebyshev spaces, Manuscripta Math. 20 (1977), 401-407.

8. R. A. Zalik, Existence of Tchebycheff extensions, J. Math. Anal. Appl. 51 (1975), 68-75.

9. R. Zielke, Alternation properties of Tchebyshev-systems and the existence of adjointed functions, J. Approx. Theory 10 (1974), 172-184.

10. D. Zwick, Characterization of WT-spaces whose derivatives form a WT-space, J. Approx. Theory 38 (1983), 188-191.

Department of Mathematics, Faculty of Education, Saga University, Saga 840, Japan 